\title{
Oxidative stress and immune cell activation quantification in sepsis and non-sepsis critical care patients by neopterin/7,8-dihydroneopterin analysis
}

https://doi.org/10.1515/pteridines-2020-0015 received February 24, 2020; accepted May 5, 2020.

\begin{abstract}
:
Introduction: Neopterin and 7,8-dihydroneopterin are used as biomarkers of oxidative stress and inflammation, but the effect of kidney function on these measurements has not been extensively explored. We examine the levels of oxidative stress, inflammation and kidney function in intensive patients and compare them to equivalent patients without sepsis.

Methods: 34 Intensive care patients were selected for the study, 14 without sepsis and 20 with. Both groups had equivalent levels of trauma, assessed by SAPS II, SOFA, and APACHE II and III scores. Plasma and urinary neopterin and total neopterin (neopterin + 7,8-dihydroneopterin) values were measured.
\end{abstract}

Results: Neopterin and total neopterin were significantly elevated in urine and plasma for multiple days in sepsis versus non-sepsis patients. Plasma neopterin and total neopterin have decreasing relationships with increased eGFR $(\mathrm{p}<0.008$ and $\mathrm{p}<0.001$, respectively).

\footnotetext{
*Corresponding author: Steven P. Gieseg, School of Biological Sciences, University of Canterbury, Christchurch, New Zealand, E-mail: Steven.Gieseg@canterbury.ac.nz

Gregory Baxter-Parker, School of Biological Sciences, University of Canterbury, Christchurch, New Zealand

Ravinder Reddy Gaddam, Division of Cardiovascular Medicine, Department of Internal Medicine, Abboud Cardiovascular Research Center, University of lowa Carver College of Medicine, lowa City, lowa, USA

Elena Moltchanova, Department of Mathematics and Statistics, University of Canterbury, Christchurch, New Zealand

Anitra Carr, Stephen Chambers, Department of Pathology \& Biomedical Science, University of Otago Christchurch, Christchurch, New Zealand Geoff Shaw, Department of Intensive Care, Christchurch Hospital, New Zealand
}

Plasma/urinary neopterin and total neopterin ratios demonstrate that total neopterin flux is more influenced by eGFR than neopterin, with significantce of $\mathrm{p}<0.02$ and $\mathrm{p}<0.0002$ respectively.

Conclusion: Sepsis patients present with greater levels of oxidative stress and immune system activation than non-sepsis patients of equal levels of trauma, as measured by neopterin and total neopterin. eGFR may need to be taken into account when accessing the level of inflammation from urinary neopterin measurements.

Keywords: Sepsis; neopterin; 7,8-dihydroneopterin; kidney function; inflammation; biomarkers.

\section{Introduction}

Sepsis is a severe, life threatening, condition caused by the body's immune system responding to a systemic infection. Approximately 30 million people worldwide are afflicted by sepsis each year, with an overall mortality rate of between $20 \%$ and $41 \%$ for the initial stage of sepsis. As sepsis progresses, mortality rates increase up to a staggering $50 \%[1,2]$. A crucial factor in reducing the risk of mortality is early detection and treatment [3]. A plethora of biomarkers have been investigated for assessing severity of sepsis patients. These include; neopterin (NP) [4], C- reactive protein (CRP) [5], procalcitonin [6] and interleukin-6 (IL-6) [7]. Recently, hydrogen sulfide and substance $P$ have also been investigated by Gaddam et. al. [8]. The presented study is a continuation of that work and has used the same patient cohorts while investigating different biomarkers. Additionally, the CRP and IL-6 data from this study has been reanalysed in respect to correlations with neopterin and total neopterin (TNP) for greater comparison between inflammation biomarkers. 
Table 1: SOFA, APACHE II and III, and SAPS II assessment characteristics and score ranges.

\begin{tabular}{|c|c|c|c|}
\hline Algorithm & Factors measured & Score range & Predictor \\
\hline SOFA & $\begin{array}{l}\text { Respiration, Glasgow Coma Scale, Mean Arterial Pressure, } \\
\text { Bilirubin, Coagulation, Plasma Creatinine. }\end{array}$ & $\begin{array}{l}0-24 \text { (daily } \\
\text { measurements) }\end{array}$ & Changing risk of mortality \\
\hline APACHE II & $\begin{array}{l}\mathrm{AaDO}_{2} \text { or } \mathrm{PaO}_{2} \text {, Temperature, Mean Arterial Pressure, } \mathrm{pH} \\
\text { arterial, Heart Rate, Respiratory Rate, Sodium, Potassium, } \\
\text { Creatinine, Haematocrit, White Blood Cell Count, Glasgow } \\
\text { Coma Scale. }\end{array}$ & $\begin{array}{l}0-71 \text { (single } \\
\text { measurement) }\end{array}$ & $\begin{array}{l}\text { Assessment of disease } \\
\text { severity }\end{array}$ \\
\hline APACHE III & $\begin{array}{l}\text { APACHE II Factors with additional calculation for ICU } \\
\text { admission condition (from a list of } 212 \text { conditions), Age, Sex, } \\
\text { Race, Pre-existing Comorbidities, Prior Location. }\end{array}$ & $\begin{array}{l}0-299 \text { (single } \\
\text { measurement) }\end{array}$ & $\begin{array}{l}\text { Assessment of disease } \\
\text { severity }\end{array}$ \\
\hline SAPS II & $\begin{array}{l}\text { Age, Heart Rate, Blood Pressure, Temperature, Glasgow Coma } \\
\text { Scale, CPAP, } \mathrm{PaO}_{2}, \mathrm{FiO}_{2} \text {, Urine Output, Blood Urea Nitrogen, } \\
\text { Sodium, Potassium, Bicarbonate, Bilirubin, White Blood Cell } \\
\text { Count, Chronic Diseases, Admission type. }\end{array}$ & $\begin{array}{l}0-163 \text { (single } \\
\text { measurement) }\end{array}$ & $\begin{array}{l}\text { Prediction for chance of } \\
\text { mortality }\end{array}$ \\
\hline
\end{tabular}

Although CRP and procalcitonin are two of the most commonly used biomarkers in sepsis research, they are unreliable at predicting clinical outcome [5]. Clinical data indicates that plasma neopterin may be useful in early detection of sepsis development after trauma or surgery, as well as sepsis progression $[9,10]$. Neopterin is an oxidation product of its parent compound, 7,8-dihydroneopterin [11-13], a monocyte and macrophage cell synthesised antioxidant [14] produced upon activation of the immune system [15]. Therefore, neopterin can be used as a biomarker for immune derived oxidative stress and 7,8-dihydroneopterin as a biomarker for monocyte and macrophage cell activation $[16,17]$. Due to limitations in detection, 7,8-dihydroneopterin is oxidised to the highly fluorescent neopterin for measurement, with the combination of neopterin and oxidised 7,8-dihydroneopterin being deemed "total neopterin" [18, 19].

Greatly elevated neopterin levels were found to be an indicator of developing septic shock , and predictor of impending mortality [20]. However, issues with neopterin may arise when being measured in only serum or urine and not both, as it is rapidly cleared from plasma into urine by the kidneys [21]. Therefore, urinary or plasma values may be affected in septic patients where reduced kidney function is common. Previous research has shown that plasma levels of neopterin are affected by glomerular filtration rate (GFR) in patients experiencing chronic renal failure [22]. However, this work investigated patients who already had kidney failure (GFR $<15 \mathrm{~mL} / \mathrm{min} / 1.73 \mathrm{~m}^{2}$ ), which does not account for patients whose kidneys are only beginning to fall below normal functionality. It may be possible that kidney function has a significant impact on plasma neopterin (pNP) and urinary neopterin (uNP) values in sepsis patients who have reduced kidney function (GFR $<60 \mathrm{~mL} / \mathrm{min} / 1.73 \mathrm{~m}^{2}$ ) without clinical presentation of kidney failure or kidney injury. This may lead to skewed results if both mediums were not measured, or if GFR was not taking into account.

In this study we have compared urinary neopterin (uNP) and plasma neopterin (pNP) and urinary total neopterin (uTNP) and plasma total neopterin (pTNP) values between critically ill patients with sepsis labelled as or an equivalent level of trauma and organ dysfunction (non-sepsis). Equivalence was achieved by measurement and matching of patients Sequential Organ Failure Assessment (SOFA), Simplified Acute Physiology Score (SAPS) II and Acute Physiology And Chronic Health Evaluation (APACHE) II and III scores (characterised in Table 1). The equivalence between the two groups was to control for any bias from condition/injury severity and organ functionality. We have measured neopterin and total neopterin to assess oxidative stress and immune system activity during septic infection. Measurements were made in plasma and urine with eGFR being monitored to determine the impact that kidney function may have on neopterin and total neopterin flux into the urine. We hypothesise that the levels of these biomarkers will be increased in sepsis patients versus non-sepsis, and decreased eGFR will cause neopterin and total neopterin to increase in plasma relative to urine. 


\section{Materials and methods}

\section{Study design and clinical cohort}

The study was conducted in the Intensive Care Unit (ICU) of Christchurch Hospital, New Zealand, in 201516. Alternatively, if informed consent was not possible, provisional consent was granted by a family member or friends and reviewed by the patients upon their recovery. Selection of septic patients was based on the study inclusion parameters for the Australasian Resuscitation in Sepsis Evaluation-Randomised Controlled Trial (ARISERCT) which can be found in detail at www.arise.org.au [23]. The sepsis cohort also included patients that had suspected or confirmed infection, systemic inflammatory response syndrome, and evidence of either refractory hypotension or hypoperfusion. Criteria for involvement of non-sepsis patients were, being over the age of 18 and not being expected to die within 72 hours. Patients admitted to the ICU that presented evidence of refractory hypotension or hypoperfusion, but not sepsis or any know inflammatory condition, were included in the non-sepsis cohort. Exclusion criteria included patients who had a contraindication to blood products, haemodynamically unstable due to bleeding, pregnant (confirmed or suspected), a bowel infarction (confirmed or suspected), and in-patient transfer from another health care facility. Patients were assessed by two independent investigators to determine eligibility for the study. Non-sepsis patients were also required SOFA, SAPS II, and APACHE II and III scores (characterised in Table 1) equivalent to that of the sepsis cohort to match levels of trauma and organ dysfunction between the groups. All patients were treated with standard supportive medicine, including; fluid resuscitation, medical and technological interventions for organ dysfunction and failure, empirical antibiotic treatment, and vasoactive drugs, in accordance with hospital practice guidelines.

Ethical approval: The research related to human use has been complied with all the relevant national regulations, institutional policies and in accordance the tenets of the Helsinki Declaration, and has been approved by the Health and Disability Ethics Committee, Wellington, New Zealand (15/SHT/36).

Informed consent: For some patients, informed consent was gathered prior to enrolment in the study. Alternatively, if informed consent was not possible, provisional consent was granted by a family member or friends and reviewed by the patients upon their recovery.

\section{Variables recorded}

Age, gender, type of infection, APACHE II and III Scores, SAPS II were all collected from patients during the study period. APACHE II and III and SAPS II scores were determined on the day of admission to ICU. SOFA scores were determined daily from days 0 to 4 .

\section{Blood and urine sampling and analysis}

Blood and urine samples were collected from all ICU patients from the time of admission to 96 hours. These samples were collected only during a patients stay in ICU, at time intervals of $0,12,24,48,72$, and 96 hours. Urine was collected into $70 \mathrm{~mL}$ urine pottles and immediately frozen in a $-20^{\circ} \mathrm{C}$ freezer. Blood samples from sepsis and non-sepsis patients were drawn through venepuncture and collected into a plastic tube containing EDTA before being placed on ice. Within 5 minutes of being placed on ice, blood samples were centrifuged at $1000 \mathrm{~g}$ for 10 minutes at $4^{\circ} \mathrm{C}$ and the plasma collected. C-reactive protein analysis was performed in accordance to the protocols at Canterbury Health Laboratory, Christchurch, New Zealand, before the remaining plasma was frozen and kept at $-80^{\circ} \mathrm{C}$ until required.

\section{Pterin determination}

High performance liquid chromatography measurements of neopterin (NP) were made using a Shimadzu 20A HPLC (Shimadzu Scientific Instruments, New Zealand) with a Sil-20A autosampler and RF-20Axls fluorescence detector. Neopterin separation for urine and plasma samples was achieved with a Luna $5 \mu \mathrm{m} \mathrm{SCX} 100 \AA 250 \mathrm{~mm} \times 4.6 \mathrm{~mm}$ column, using $20 \mathrm{mM}$ ammonium phosphate $\mathrm{pH} 2.5$ and a mobile phase being pumped at $1.0 \mathrm{ml} \mathrm{min}^{-1}$ for 12 minutes per sample [18]. Fluorescence wavelengths for neopterin detection were set at 438 and $353 \mathrm{~nm}$ for emission and excitation, respectively. Peak analysis and integration was performed by Shimadzu Lab Solutions version 5.86 software.

For analysis of total neopterin (TNP) both urine and plasma, $20 \mu \mathrm{L}$ of acidic iodide $\left(5.4 \% \mathrm{I}_{2} / 10.8 \% \mathrm{KI}\right.$ in $1 \mathrm{M}$ $\mathrm{HCl})$ was added to $100 \mu \mathrm{L}$ of urine or protein precipitated plasma, before being vortexed and left in the dark at room temperature to oxidise the 7,8-dihydroneopterin into neopterin. After 15 minutes of incubation, $10 \mu \mathrm{L}$ of $0.6 \mathrm{M}$ ascorbic acid was added, followed by vortexing. $100 \mu \mathrm{L}$ of 
this solution was then transferred into HPLC vials for total neopterin analysis [18].

\section{Urine analysis and specific gravity}

$5 \mu \mathrm{L}$ of defrosted urine was vortexed with $195 \mu \mathrm{L}$ of 20 $\mathrm{mM}$ ammonium phosphate ( $\mathrm{pH} 2.5$ ) before $100 \mu \mathrm{L}$ was transferred into high-performance liquid chromatography (HPLC) vials for urinary neopterin (uNP) analysis. The remaining $100 \mu \mathrm{L}$ was used for acidic iodide treatment as described above to measure urine total neopterin (uTNP).

Specific gravity (SG) was measured using an ATAGO $\mathrm{N}-20$ refractometer, and calculated as described previously [18], using the formula described below and based on the normal population $\mathrm{SG}_{1.020}$ [24]. Before measurement, samples were brought to room temperature to remove and temperature-dependent density variation bias [25].

$$
[\mathrm{uNP}]\left(\mathrm{nM} / \mathrm{SG}_{1.020}\right)=\left(\mathrm{SG}_{1.020}-1\right) /\left(\mathrm{SG}_{\text {sample }}-1\right) \times[\mathrm{uNP}](\mathrm{nM})
$$

\section{Plasma neopterin}

$100 \mu \mathrm{L}$ of defrosted plasma was vortexed thoroughly with $100 \mu \mathrm{L}$ of acetonitrile before being centrifuged at $21,000 \mathrm{~g}$ for 5 minutes at $4^{\circ} \mathrm{C} .100 \mu \mathrm{L}$ of the supernatant was transferred to HPLC vials for plasma neopterin (pNP) analysis. The remaining $100 \mu \mathrm{L}$ was treated with acidic iodide to convert the 7,8-dihydroneopterin to neopterin before HPLC analysis for plasma total neopterin (pTNP).

\section{Creatinine and eGFR determination}

Plasma creatinine levels were determined as part of a standard plasma medical test by Canterbury Health Laboratories, Christchurch, New Zealand. The method used was a classical Jaffe Reaction with an Abbot c series analyser and Abbot reagents. Blood specimens were collected in either $4.5 \mathrm{~mL}$ Vacutainer (Heparinised) PST Tubes, vacutainer SST (clotting) tubes or plain vacutainer tubes.

The CKD-EPI (Chronic Kidney Disease Epidemiology Collaboration) equation [26] was used to determine eGFR from serum creatinine concentration.

This formula is:

$\mathrm{eGFR}=141 \mathrm{x} \min (\mathrm{SCr} / \mathrm{k}, 1)^{\mathrm{a}} \mathrm{x} \max (\mathrm{SCr} / \mathrm{k}, 1)^{-1.209} \mathrm{x} 0.993^{\mathrm{Age}} \mathrm{x}$ [1.018 if Female] $\mathrm{x}$ [1.159 if Black]
$\mathrm{SCr}$ is serum creatinine, $\mathrm{k}$ is 0.7 for females and 0.9 for males, a is -0.329 for females and -0.411 for males. Min indicates the minimum $\mathrm{SCr} / \mathrm{k}$ or 1 , max indicates the maximum $\mathrm{SCr} / \mathrm{k}$ or 1 . Age is in years.

\section{IL-6 determination}

Plasma interleukin-6 (IL-6) levels were measured using enzyme-linked immunosorbent assay (ELISA) DuoSet kits from R\&D Systems (Minneapolis, USA) in accordance with the manufacturer's protocol. ELISA specific plates were coated with capture antibody and incubated overnight at room temperature. Plates were then aspirated, washed with phosphate buffered saline-tween (PBST, 0.05\% w/v) and blocked with $300 \mu \mathrm{L}$ of bovine serum albumin (BSA, $1 \%$ $\mathrm{w} / \mathrm{v}$ ) for 1 hour at room temperature, followed by washing and addition of $100 \mu \mathrm{L}$ plasma sample or standard. After 2 hours of incubation, plates were decanted, washed and $100 \mu \mathrm{L}$ of biotin-conjugated detection antibody was added. After 2 hours plates were decanted, washed and $100 \mu \mathrm{L}$ of streptavidin-conjugated HRP was added. Following a 20 minute incubation, plates were decanted, washed and 100 $\mu \mathrm{L}$ of substrate reagent was added. Plates were incubated at room temperature for up to 20 minutes to allow the colour to develop before the reaction was terminated by addition of $50 \mu \mathrm{L}$ of $\mathrm{H}_{2} \mathrm{PO}_{4}$. Plates were read at $450 \mathrm{~nm}$ on a spectrophotometer (Spectramax, Molecular Devices, USA) with a $570 \mathrm{~nm}$ correction.

\section{Statistical analysis}

A repeated measures 2-way ANOVA was performed to test the effects of group and hours on the biomarker levels. To account for repeated measures, mixed effect regression models were fitted to test the effect of eGFR on neopterin and total neopterin, as well as to study the correlation between creatinine and neopterin. The response variables were logged to improve the normality and heteroscedasticity of the residuals and of the random effects, and the models were checked with respect to these diagnostics. Bootstrap methods were used where the assumptions did not apply [27]. All modelling was done using the R-software packages lme4, nlme and boot . For the presented results, * denotes statistical significance between non-sepsis and sepsis groups. Graphical figures were generated using R and GraphPad Prism 7.0. Figures were produced using GraphPad Prism version 6.0 or R. 


\section{Results}

\section{Demographics of patients}

A total of 34 patients were recruited for this study. Of this cohort, non-sepsis patients made up 14 of the subjects, with sepsis making up the remaining 20 patients (Table 2). There was no significant difference in mean age between these two groups, but there was dominance towards male patients (10 and 12 for non-sepsis and sepsis, respectively). Five of the patients died (three from non-sepsis and two with sepsis) during the duration of their time in ICU while on the study. There was a slight predominance of males in the study, however, this was not considered to introduce any bias due to the lack of significant difference for neopterin or 7,8-dihydroneopterin production between genders [28].

\section{Comparison of organ dysfunction and disease severity between sepsis and non-sepsis}

There was no statistical difference between the sepsis and non-sepsis groups for SAPS II and APACHE II and III. Likewise, SOFA scores were not statistically different on any day (Table 3). Overall, during the 5 days of patient monitoring in ICU, mean SOFA scores decreased by $22.8 \%$ and $30.8 \%$ for non-sepsis and sepsis groups, respectively.

\section{Urinary and plasma neopterin and total neopterin levels in sepsis and non-sepsis patients}

Urinary neopterin (uNP) and urinary total neopterin (uTNP) levels were determined in the non-sepsis and sepsis patients (Fig. 1) for each time point in the study (0, 12, 24, 48, 72 and 96 hours). uNP and uTNP are significantly elevated over non-sepsis at 0,12, 24, 48 and 72 hours for uNP, and 0, 12, 24 and 48 hours for uTNP. There is a mean uNP decreases in the sepsis cohort by $46 \%$ over the study period while increasing by $32 \%$ in the non-sepsis group. Likewise, mean uTNP decreases by $65 \%$ for sepsis patients and increases by 39\% for non-sepsis over the 96 hours. These results represent a trend but are not statistically significant due to high drop off rates at later time points.

Plasma neopterin (pNP) and plasma total neopterin (pTNP) levels were also determined in sepsis and nonsepsis patients (Fig. 2) over the 96 hour study period.

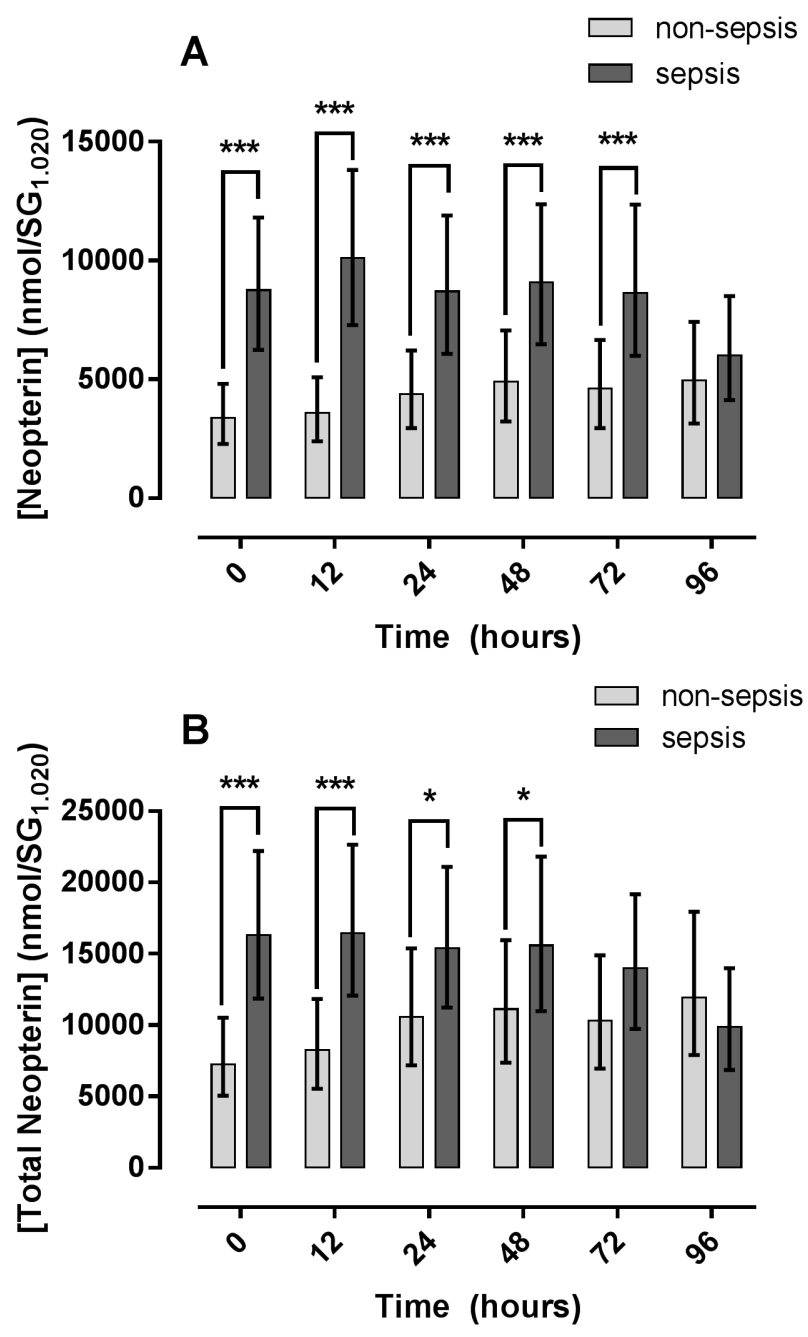

Figure 1: Urinary neopterin (A) and total neopterin (B) of non-sepsis and sepsis patients over 96 hours. Sepsis urinary neopterin levels are significantly elevated over non-sepsis up to 72 hours. Urinary total neopterin (UTNP) is elevated for the first 48 hours for sepsis versus non-sepsis. Data is presented as means and their respective $95 \% \mathrm{Cl}$.

Sepsis pNP is highly elevated over non-sepsis at every time point. pTNP is significantly higher in sepsis than nonsepsis for the first 48 hours. There was a mean decrease in pNP of $25 \%$ for sepsis patients and $14 \%$ for non-sepsis. pTNP decreases by $34 \%$ for sepsis, whereas there is a $24 \%$ increase seen in non-sepsis patients.

The TNP over NP ratios in both urine and plasma were calculated to determine the level of monocyte and macrophage activation compared to oxidative stress (Fig. 3). The TNP/NP ratio is higher for non-sepsis patients in both urine and plasma at every time point, significantly so at 24 hours in plasma. 
Table 2: Characteristics of sepsis and non-sepsis patients admitted to the medical intensive care unit during the 2015-16 study period. Some sepsis patients had more than one condition or microorganism present.

\begin{tabular}{|c|c|c|}
\hline & Non-sepsis & Sepsis \\
\hline Number of patients & 14 & 20 \\
\hline Age (Mean \pm SD) & $62.79 \pm 11.27$ & $63.28 \pm 14.34$ \\
\hline Male & 10 & 12 \\
\hline Female & 4 & 8 \\
\hline \multicolumn{3}{|l|}{ Primary criteria for ICU admission } \\
\hline Post-coronary artery by-pass grafting & 4 & \\
\hline Post-repair of aortic dissection & 2 & \\
\hline Persistent seizures causing multiple fractures and soft tissue injury & 2 & \\
\hline Post-surgery aortic stenosis & 1 & \\
\hline Cardiac arrest out of hospital causing brain injury & 1 & \\
\hline Myocardial infarction & 1 & \\
\hline Congestive heart failure & 1 & \\
\hline Failed renal transplant & 1 & \\
\hline Car crash causing multiple trauma & 1 & \\
\hline Peritonitis & & 7 \\
\hline Pneumonia & & 6 \\
\hline Cholecystitis and pancreatitis & & 1 \\
\hline Fourniers gangrene & & 1 \\
\hline Necrotising pancreatitis & & 1 \\
\hline Multiple organ failure & & 1 \\
\hline Osteomyelitis of ischium & & 1 \\
\hline Perforated appendix & & 1 \\
\hline Perforated sigmoid colon & & 1 \\
\hline Pyelonephritis & & 1 \\
\hline Septicaemia & & 1 \\
\hline Septic arthritis & & 1 \\
\hline \multicolumn{3}{|l|}{ Microorganism } \\
\hline Polymicrobial & & 8 \\
\hline Unknown & & 7 \\
\hline Streptococcus pneumonia & & 3 \\
\hline Cytomegalovirus & & 1 \\
\hline Klebsiella pneumonia & & 1 \\
\hline Pantoea agglomerans & & 1 \\
\hline Polymicrobial + Pseudomonas aeruginosa & & 1 \\
\hline Stenotrophomonas maltiphilia & & 1 \\
\hline
\end{tabular}


Table 3: SOFA, SAPS II, and APACHE II and III clinical scoring for nonsepsis and sepsis patients admitted to the medical ICU during the 2015-16 study period. There is no statistically significant difference between non-sepsis and sepsis patients for any assessment score.

\begin{tabular}{lll}
\hline & Non-sepsis & Sepsis \\
\hline & $($ Mean \pm SD) $(\mathrm{n})$ & $($ Mean \pm SD) $(\mathrm{n})$ \\
& SOFA Score & SOFA Score \\
Day & All patients & All patients \\
0 & $10.1 \pm 2.3(14)$ & $9.1 \pm 2.6(20)$ \\
1 & $9.6 \pm 3.5(14)$ & $8.1 \pm 3.0(15)$ \\
2 & $9.2 \pm 3.4(14)$ & $7.0 \pm 3.5(13)$ \\
3 & $8.5 \pm 3.2(11)$ & $5.5 \pm 3.1(12)$ \\
4 & $7.8 \pm 4.5(9)$ & $6.3 \pm 4.9(7)$ \\
& SAPS II & \\
& $46.4 \pm 12.5(14)$ & $43.8 \pm 17.3(19)$ \\
& APACHE II & \\
$21.2 \pm 7.0(14)$ & \\
& APACHE III & \\
$78.2 \pm 23.1(14)$ &
\end{tabular}

\section{Effect of eGFR on plasma and urinary neopterin and total neopterin levels for sepsis patients}

Urinary and plasma measurements of neopterin or 7,8-dihydroneopterin are often made without consideration of the effect that kidney function may have. In this study we have made these measurements in both plasma and urine while also analysing the effect of kidney function. Estimated glomerular filtration rate (eGFR) for sepsis patients was calculated and compared to pNP, uNP, pTNP and uTNP levels to investigate how kidney function effects these biomarkers flux from plasma into urine (Fig. 5). Both pNP and pTNP have statistically significant decreasing relashionships with the increasing rate of eGFR $(p<0.008$ and $\mathrm{p}<0.001$, respectively), whereas $\mathrm{UNP}$ and $\mathrm{uTNP}$ remain relatively flat. pNP (Fig. $5 \mathrm{~A}$ ) decreases as eGFR increases at a rate of $\mathrm{Y}=103.2-0.6 \mathrm{x}$ eGFR. Although uNP (Fig. $5 \mathrm{~B}$ ) significantly correlates to eGFR $(\mathrm{p}<0.009)$ it is not largely effected by the value of eGFR ( $\mathrm{Y}=10854.7+4.1 \mathrm{x}$ eGFR). pTNP (Fig. 5 C) significantly ( $<<0.001)$ correlates to eGFR, with decreasing $\mathrm{pNP}$ values as eGFR increases $(\mathrm{Y}=285.0$ $1.8 \mathrm{x}$ eGFR). uTNP does not significantly correlate to eGFR but has a very minor decreasing effect with increase eGFR $(\mathrm{Y}=20261.3-27.5 \mathrm{x}$ eGFR).
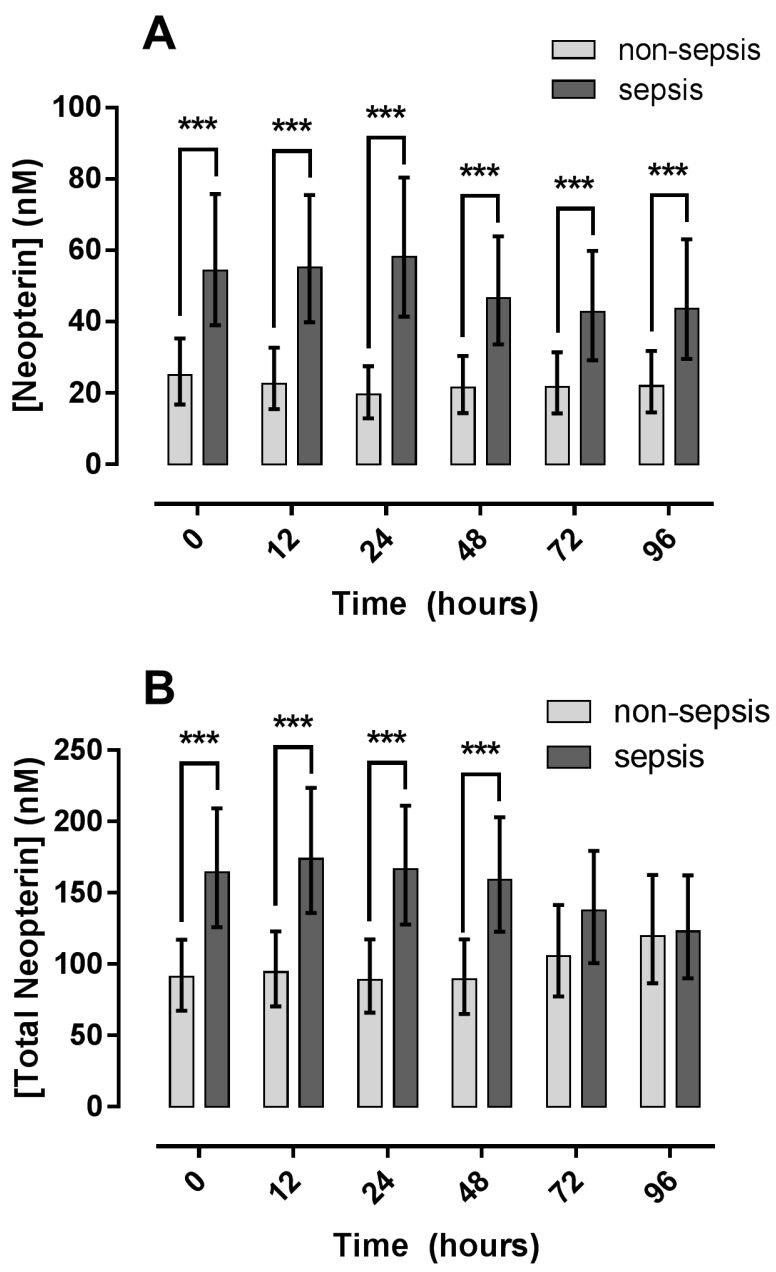

Figure 2: Plasma neopterin (A) and total neopterin (B) for nonsepsis and sepsis patients for 96 hours following admission to ICU. Sepsis plasma neopterin (pNP) is highly significantly elevated over non-sepsis for all time points. Sepsis total neopterin (pTNP) is significantly elevated for 48 hours, but is equal to non-sepsis by 96 hours. Data is presented as means and their respective $95 \% \mathrm{Cl}$.

As the results above are assessed as grouped data with repeated measures, it does not take individuals into account. To assess the effect of eGFR on individual patients, ratios of the biomarkers between urine and plasma were calculated against eGFR for each patient (Fig. 6). The data was calculated for each time point in which both biomarkers and eGFR were obtained, with raw data being used for urinary levels. Neopterin (Fig. 6 A) shows a statistically significant $(\mathrm{p}<0.02)$ increasing trend $(\mathrm{Y}=91.0+2.1 \mathrm{x}$ eGFR) towards being relatively more present in urine rather than plasma with increased eGFR. At the lower end eGFR $\left(25 \mathrm{~mL} / \mathrm{min} / 1.73 \mathrm{~m}^{2}\right)$, neopterin concentration in urine is 143 times that of plasma, whereas, at the higher end of eGFR $(100 \mathrm{~mL} / \mathrm{min} / 1.73$ $\mathrm{m}^{2}$ ) urinary neopterin concentration is 301 times larger 

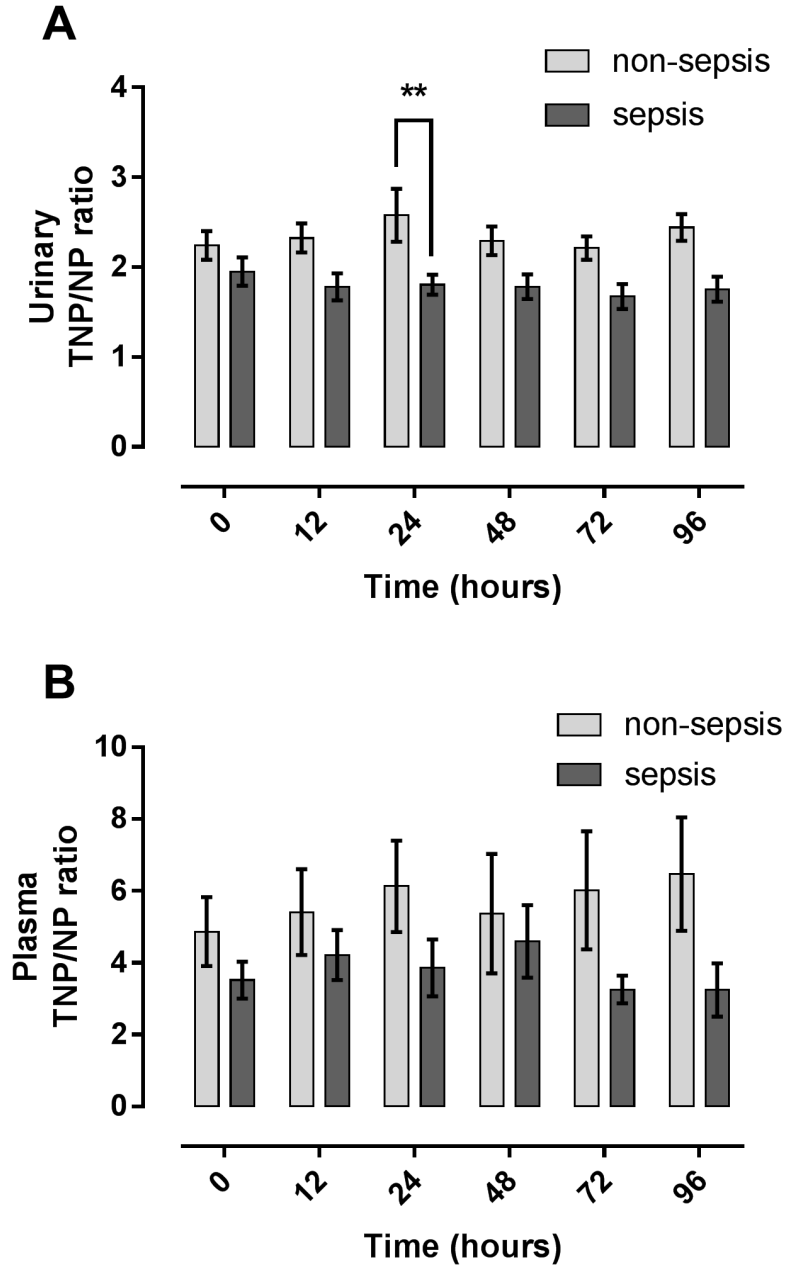

Figure 3: Urinary (A) and plasma (B) total neopterin to neopterin ratios. Sepsis patients demonstrate a trend of lower TNP/NP ratios in both urine and plasma over all time points, significantly so in urine at 24 hours. Data is presented as means with $95 \% \mathrm{Cl}$

than plasma. Total neopterin (Fig. 5 B) shows a stronger, statistically significant $(\mathrm{p}<0.0002)$, positive trend $(\mathrm{Y}=35.6$ $+1.4 \mathrm{x}$ eGFR $)$ with the lower end eGFR $(25 \mathrm{~mL} / \mathrm{min} / 1.73$ $\mathrm{m}^{2}$ ) having 70 times the concentration of total neopterin in urine compared to plasma. On the higher end eGFR (100 $\mathrm{mL} / \mathrm{min} / 1.73 \mathrm{~m}^{2}$ ), total neopterin concentrations are 173 times higher in urine. This is 2.1 and 2.5 times differences between lower and higher eGFR range for neopterin and total neopterin, respectively.

pNP and pTNP were analysed against serum creatinine (Fig. $7 \mathrm{~A}$ and B). pNP correlates strongly to plasma creatinine $(p<0.0001)$ with a positive trend $(Y=28.7+0.3 x$ creatinine). pTNP also correlates strongly $(\mathrm{p}<0.0001)$ with plasma creatinine and shows a positive trend $(\mathrm{Y}=91.6+$ $0.8 \times$ creatinine).

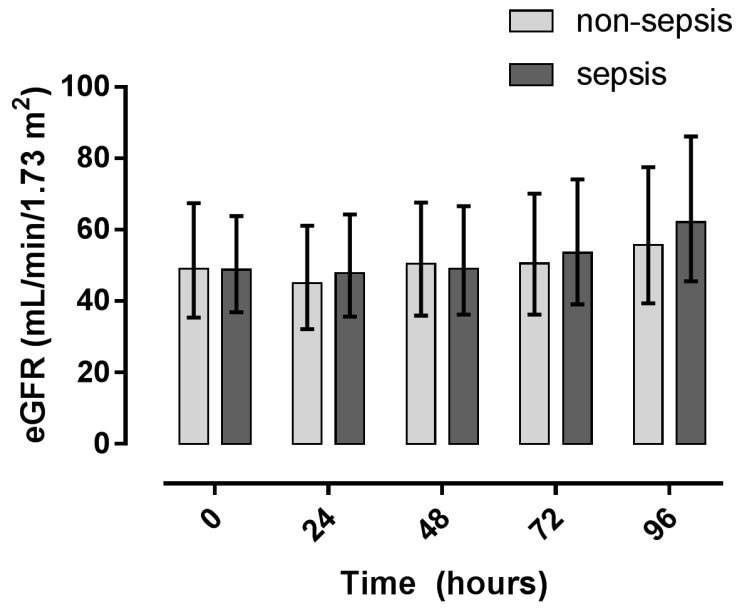

Figure 4: eGFR measurements for non-sepsis and sepsis patients. There are no statistically significant differences between the groups at any time. The data is presented as means with their respective $95 \% \mathrm{Cl}$.

\section{IL-6 and CRP in non-sepsis and sepsis patients against neopterin values}

In this study we have investigated correlations between plasma IL- 6 and CRP against NP and TNP from plasma and urine (Table 4). CRP levels are significantly higher in the sepsis group at time 0, 24 and 48 hours, while IL-6 remains elevated in sepsis patients until 72 hours. In sepsis patients, IL-6 shows almost no correlation to NP or TNP levels from urine or plasma. For non-sepsis IL-6 values, there is an extremely weak correlation to pTNP ( $\mathrm{r}$ $=0.2572\left(^{\star}\right)$ ). Likewise, sepsis CRP values do not correlate to NP or TNP from plasma or urine. UNP and uTNP show moderate correlations to non-sepsis CRP values of $\mathrm{r}=$ $\left.0.4912^{(\star \star \star}\right)$ and $\left.0.3354^{(\star \star}\right)$, respectively.

\section{Discussion}

The results from this study show that sepsis significantly up-regulates the immune system over non-sepsis patients that are experiencing equivalent levels of trauma and organ dysfunction. This was demonstrated by neopterin and total neopterin (neopterin $+7,8$-dihydroneopterin) levels being elevated over non-sepsis in plasma and urine. This observation is further enhanced by CRP and IL-6 levels being elevated in the sepsis group versus nonsepsis.

There was a wide range of traumas in the non-sepsis group, with post-coronary artery by-pass being slightly 
A

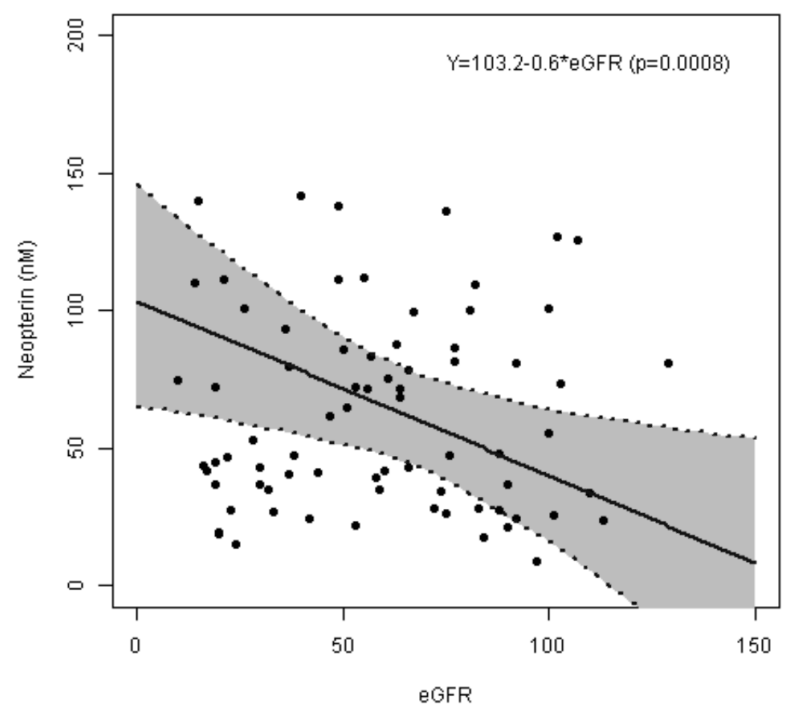

C

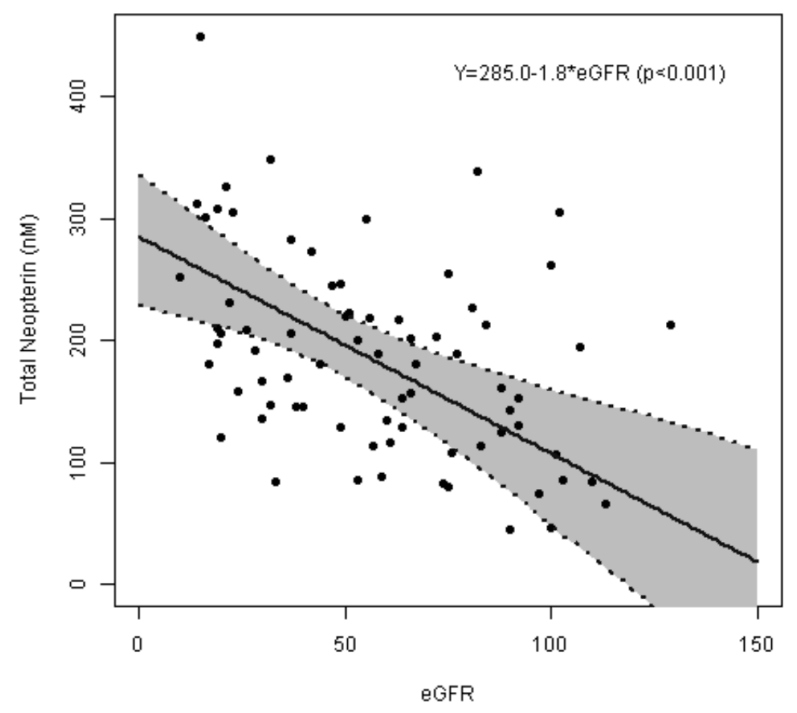

B

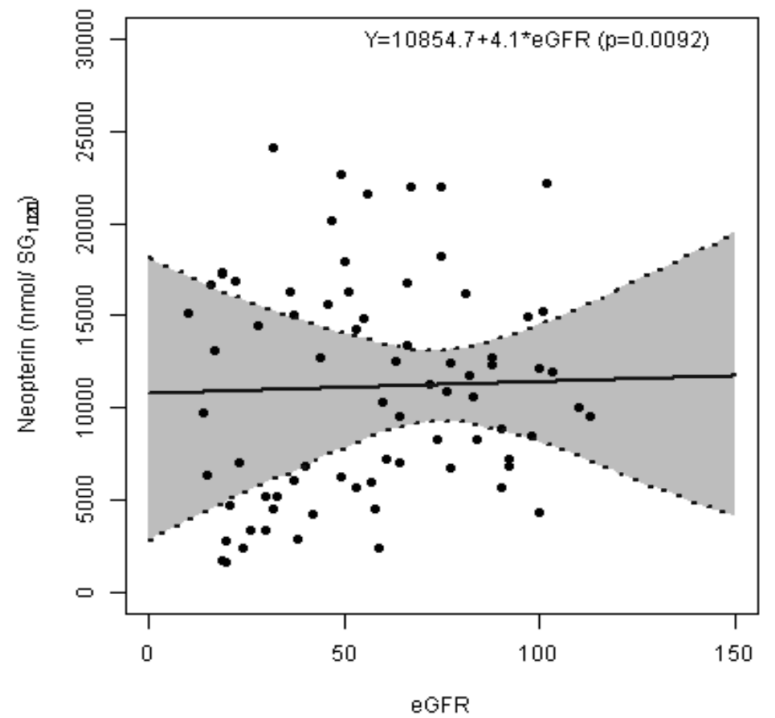

D

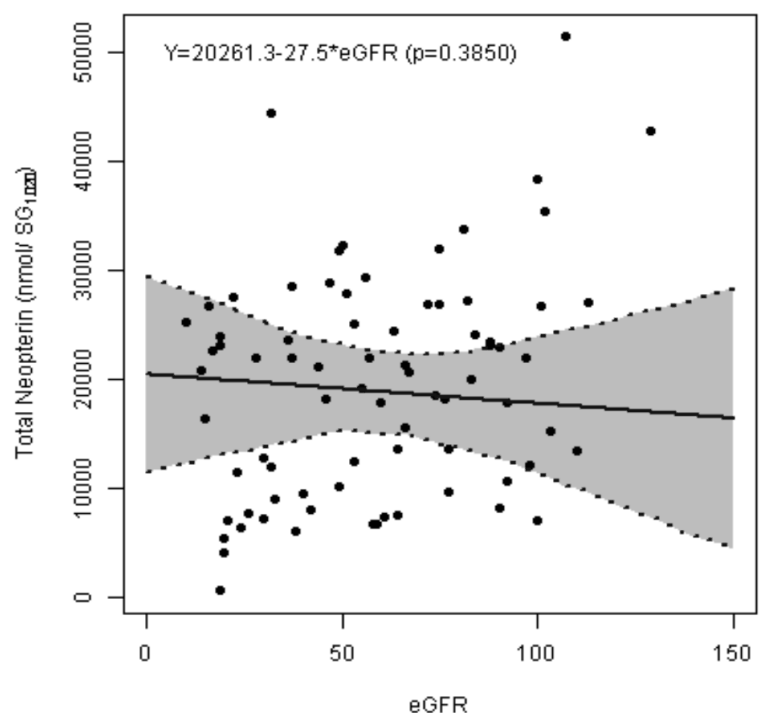

Figure 5: Plasma (A, C) and urinary (B, D) neopterin (A, B) and total neopterin (C, D) correlations against eGFR in septic patients. Plasma neopterin and total neopterin decrease as eGFR increases, whereas, urinary neopterin and total neopterin remain less effected by kidney function.

predominant. Polymicrobial and unknown organisms made up the bulk of infecting microorganisms in the sepsis group, with some patients experiencing two traumas and infections. Peritonitis and pneumonia being were the most common admission conditions. In spite of this diversity, the non-sepsis and sepsis patients in the study were experiencing an equivalent level of trauma, as measured by SOFA, SAPS-II, and APACHE II and III. These scores are used to quantify trauma, assess patient condition and predict the likelihood of organ failure and mortality [29-32]. Multiple scoring systems were chosen to minimise biases from any individual algorithm. The lack of significant difference between the non-sepsis and sepsis indicates that the equivalence of patient trauma and organ dysfunction that was aimed for was achieved.

uNP and uTNP levels can be determined accurately and rapidly with low detection limits using SCX-HPLC, allowing for potential use in a hospital setting [18, 33]. In this study, uNP and uTNP levels for sepsis were significantly elevated over non-sepsis for the first 72 and 48 hours, respectively. Following this, the increasing levels of non-sepsis neopterin and total neopterin, combined 
Table 4: Plasma IL-6 and CRP values are correlated against urinary and plasma neopterin and total neopterin values. Daily CRP and IL-6 values are shown for non-sepsis and sepsis groups with standard deviation. Significance values are compared between non-sepsis and sepsis values for the corresponding biomarker. Plasma CRP values are measured as $\mathrm{mg} / \mathrm{L}$ and plasma IL-6 is measured as $\mathrm{ng} / \mathrm{mL}$. Correlations were determined using individual data and are displayed as r values with significances. ${ }^{*}<0.05,{ }^{* *} \mathrm{p}<0.01,{ }^{* *} \mathrm{p}<0.001, \mathrm{~ns}=\mathrm{not}$ significant. The plasma CRP and IL-6 data is supporting data reanalysed from Gaddam et. al. (2017), using the same patient cohorts [7*\}.

\begin{tabular}{|c|c|c|c|c|}
\hline Time (hours) & Non-sepsis (CRP) & Sepsis (CRP) & Non-sepsis (IL-6) & Sepsis (IL-6) \\
\hline 0 & $138.4(98.4)$ & 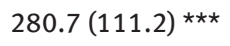 & $162.0(118.9)$ & $1410.2(2114.9) * \star \star$ \\
\hline 24 & $188.9(93.8)$ & $293.2(111.5) * \star \star$ & $87.7(57.6)$ & 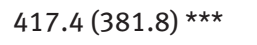 \\
\hline 48 & $207.9(107.5)$ & $221.2(105.3)$ * & $54.8(39.5)$ & 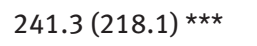 \\
\hline 72 & $181.3(111.3)$ & $188.1(101.2)$ & $75.6(113.1)$ & $209.8(285.5)$ ** \\
\hline 96 & $203.2(105.1)$ & $148.3(90.6)$ & $121.3(158.5)$ & $1052.2(2076.2)$ \\
\hline \multicolumn{5}{|l|}{ Correlations } \\
\hline uNP & $0.4912(\star \star \star)$ & -0.1966 (ns) & $-0.1021(\mathrm{~ns})$ & -0.0200 (ns) \\
\hline UTNP & $0.3354(\star \star)$ & $-0.1368(\mathrm{~ns})$ & -0.2081 (ns) & 0.0353 (ns) \\
\hline $\mathrm{pNP}$ & -0.0203 (ns) & 0.0125 (ns) & 0.1441 (ns) & -0.0407 (ns) \\
\hline pTNP & 0.1284 (ns) & 0.1828 (ns) & $0.2572(*)$ & 0.0353 (ns) \\
\hline eGFR & $-0.0933(\mathrm{~ns})$ & $-0.1958(n s)$ & 0.0335 (ns) & $-0.1399(n s)$ \\
\hline
\end{tabular}

A

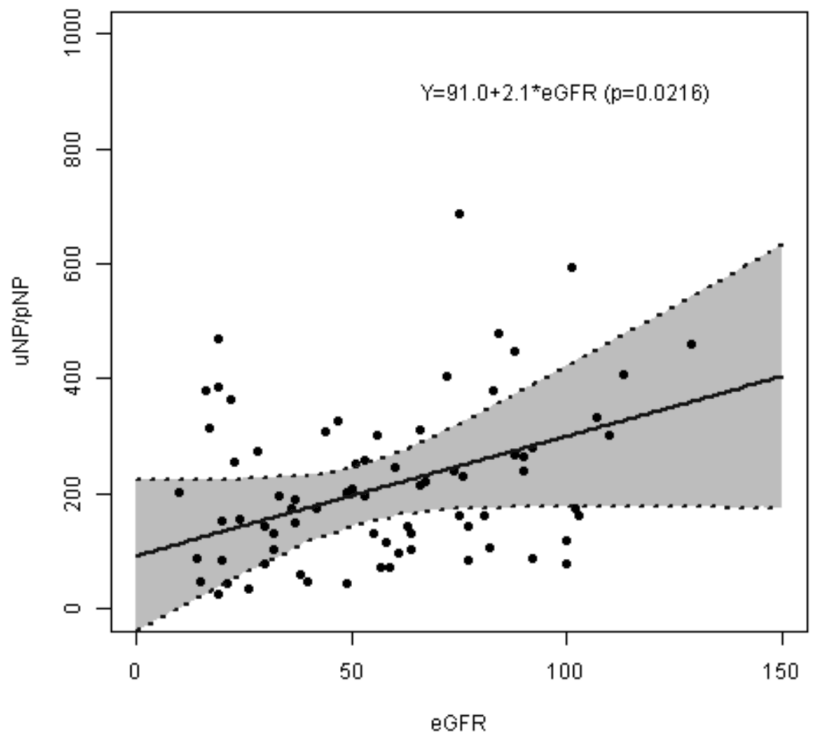

B

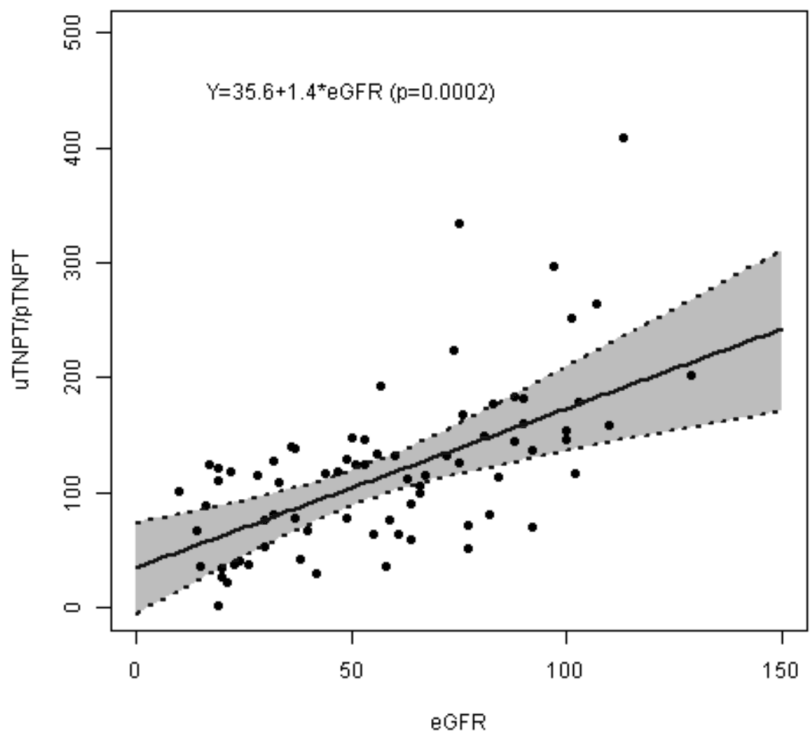

Figure 6: UNP over pNP correlation with eGFR (A) and UTNP over pTNP correlation with eGFR (B) for sepsis patients. The uNP over pNP ratio significantly correlates to eGFR ( $p<0.02$ ) with an increasing ratio to increasing eGFR trend. UTNP over pTNP significantly correlates to eGFR $(p<0.0002)$ with the trend demonstrating an increased ratio with increasing eGFR

with the decreases in sepsis equalised the biomarker levels. uNP being elevated in sepsis patients over controls is consistent with the literature [34]. uTNP has been considerably less explored throughout the literature, but here it indicates that monocyte and macrophage activation is raised considerably above the non-sepsis group at early stages. By 96 hours non-sepsis total neopterin was higher than the sepsis group, this may be due to the extensive and on-going inflammation that is subject to surgical procedures or tissue trauma [35]. pTNP loosely mimics the urinary results, with a large statistical difference for 48 hours and equalisation by 96 hours. However, uTNP levels 
A

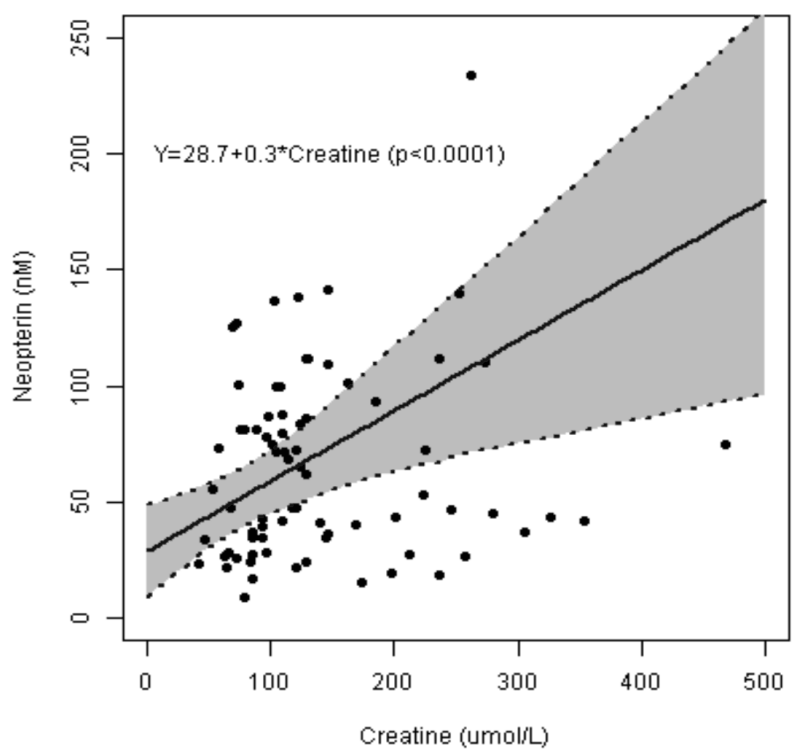

B

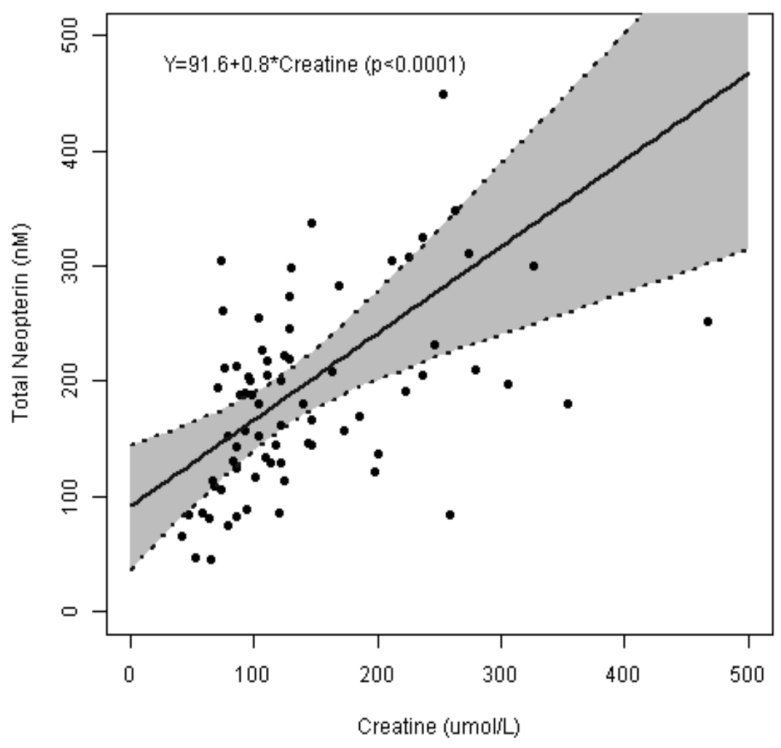

Figure 7: Plasma neopterin (A) and plasma total neopterin (B) correlated with plasma creatinine of sepsis patients. Both neopterin $(p<0.0001)$ and total neopterin $(p<0.0001)$ strongly correlated to plasma creatinine with positive trends.

increased daily in the urinary results, whereas, the plasma results showed no change until the final time points $(72$ and 96 hours). Contrary to urinary measurements, sepsis pNP remains highly elevated over non-sepsis for the duration of analysis. Many papers which have investigated neopterin or total neopterin in sepsis patients have done so by looking at either, plasma, serum, or urine, but not in unison $[4,9,34,36,37]$. It may be possible for bias to be introduced when only assessing one body fluid, particularly with the effect of kidney function discussed below. Moreover, 7,8-dihydroneopterin has been shown to be more stable when in urine than plasma $[33,38]$.

Total neopterin over neopterin (TNP/NP) ratios were determined for sepsis and non-sepsis patients. This measure gives a basic indication of monocyte and macrophage activation (TNP) versus oxidative stress (NP), e.g. a high ratio indicates significant macrophage and monocyte activation but with less oxidative stress, whereas, a low ratio indicates cell activation with large amounts of oxidative stress [39]. At every time point for urine and plasma the TNP/NP ratio was higher in non-sepsis than sepsis, indicating that there is more 7,8-dihydroneopterin present in the plasma and urine of non-sepsis patients. These results indicate the non-sepsis patients are experiencing less oxidative stress relative to sepsis patients, who had lower ratios. Combined with the overall lower TNP levels of non-sepsis patients, this data shows that the level of immune system activation and oxidative stress is significantly higher in sepsis patients, which is in agreement with the literature $[4,36,40]$. The source of oxidative stress mediated neopterin generation may be, at least in part, due to high levels of neutrophil activation found in sepsis [41]. Neutrophils produce both superoxide through the action of NADPH-oxidase, and hypochlorite by the action of myeloperoxidase. Both oxidants rapidly react with 7,8-dihydroneopterin to generate neopterin so protecting the cell from oxidative damage [11-13]. The TNP/NP ratio is doubled in plasma compared to urine, indicating an increased presence of 7,8-dihydroneopterin in a patients plasma.

CRP and IL-6 are frequently used as biomarkers in sepsis research due to their association with activation of the immune system and immune response [42-44]. CRP is produced in hepatocytes following stimulation by IL-6 [45], which is secreted by activated macrophages [46]. In this study we have shown no correlation between plasma CRP and IL-6 with plasma or urinary neopterin and total neopterin for sepsis patients. This was unexpected given the link these biomarkers have to monocyte and macrophage cell activity $[18,47]$. The lack of correlation may be due to the differences in kinetics between neopterin and 7,8-dihydroneopterin versus that of CRP, which can take up to 24 hours to change, when the body is responding to infection [42]. The increased rate of change in neopterin concentration in response to infection may be why its use has been suggested to be beneficial over 
CRP as an early warning biomarker for sepsis and septic infection following trauma [4, 9, 34]. Interestingly, there is a mild correlation between uNP and uTNP in urine, but not plasma, with CRP for the non-sepsis patients. This observation supports the validity of other clinical trials which have used UNP and uTNP as biomarkers of oxidative stress and inflammation following various forms of physical trauma $[18,19,39,48]$. Coupled with the rapid renal clearance of neopterin and 7,8-dihydroneopterin, this observation also suggests that urinary measurement of these biomarkers may be more effective than plasma measurements for determining oxidative stress and immune system activation levels in trauma patients.

With time spent in ICU, the levels of non-sepsis patients NP and TNP in both urine and plasma increased, in spite of a decrease in daily SOFA scores. The SOFA algorithm takes multiple factors into account (Table 1), and although none of these are directly related to inflammation it is not unreasonable to expect the inflammatory process to have an impact on them. Many of the non-sepsis patients had undergone surgery, which has been shown to increase inflammatory markers for an extended period of time, whilst not having a drastic impact on the factors used in generating SOFA scores $[49,50]$. This may elucidate why the non-sepsis SOFA scores decrease in spite of increased inflammatory biomarkers. In juxtaposition, uNP, pNP, uTNP, pTNP, IL-6 and CRP from patients in the sepsis group showed an overall decrease, which paralleled the improvements in daily SOFA scores. Unlike many of the conditions seen in the non-sepsis patients, sepsis is directly related to up-regulation of immune system and inflammation, which typically resolves with successful treatment [8]. Moreover, sepsis is a severe illness with systemic consequences which affects multiple systems and factors, including the ones that are used to generate SOFA scores. Taken as a whole, the results indicate that SOFA alone may is not sufficient for assessment of a patient's immune activity and can be complemented with inflammatory biomarkers.

Several studies have investigated the effect of kidney function on neopterin flux out of plasma, however, none of these studies looked at total neopterin or eGFR rates that were above renal failure levels $\left(<15 \mathrm{~mL} / \mathrm{min} / 1.73 \mathrm{~m}^{2}\right)$, nor did they measure the compounds in both plasma and urine [21, 22]. In this work, we have measured neopterin and total neopterin in plasma and urine, in conjunction with kidney functionality of wider ranges than previously investigated. The results indicate that eGFR has a moderately significant effect on neopterin flux into urine, and a highly significant effect on total neopterin, indicating that the level of 7,8-dihydroneopterin flux may be more effected by kidney function than neopterin. Alternatively, this observation may be an artefact of 7,8-dihydroneopterin oxidation in the kidney. In comparison, eGFR did not have any correlation to the levels of plasma CRP and IL-6 values for sepsis or non-sepsis patients, likely due to these compounds not being filtered by the kidneys. Using the line slope calculations, the uNP over pNP ratio was estimated to be 2.1 times higher at $\mathrm{eGFR}=100 \mathrm{~mL} / \mathrm{min} / 1.73$ $\mathrm{m}^{2}$ than $\mathrm{eGFR}=25 \mathrm{~mL} / \mathrm{min} / 1.73 \mathrm{~m}^{2}$. When applied to pTNP and uTNP, this difference was 2.5 times greater at high eGFR, again implying that total neopterin levels are more affected than neopterin by the rate at which the kidneys are filtering. Additionally, the urinary over plasma ratios of neopterin and total neopterin were found to be greater for neopterin than total neopterin overall. As total neopterin is neopterin and 7,8-dihydroneopterin combined, the results infer that it is the 7,8-dihydroneopterin portion leading to the imbalance.

Plasma creatinine, which is used to generate eGFR values, correlates strongly to pNP and even more strongly to pTNP. This would be expected to coincide with neopterin and total neopterin retention, as the decrease of kidney function is associated with the increase of plasma creatinine [51]. The same effect has been seen when measuring pNP and uNP after kidney transplantation due to end-stage renal failure [48]. The steepness of the total neopterin line $(\mathrm{Y}=91.6+0.8 \mathrm{x}$ creatinine) is greater than that of the neopterin line $(\mathrm{Y}=28.7+0.3 \times$ creatinine $)$, again indicating that 7,8-dihydroneopterin level are more affected by kidney function than neopterin.

There are two possible hypotheses for this observation: firstly, the 7,8-diydroneopterin is being reabsorbed via an undiscovered mechanism, or alternatively, that the 7,8-dihydroneopterin is being oxidised between being in the plasma and being excreted into urine. The presented data cannot determine between the two but overall the evidence leans towards the oxidation theory, due to the oxidative environment generated within kidney tissue under many illness conditions [52]. Nevertheless, our work does highlight the significance of eGFR on the observable biomarker levels and shows a potential for skewed results when kidney function is not taken into consideration in critically ill patients. Standardisation of uNP and uTNP by specific gravity has been compared to creatinine and validated [18], our results also indicate that this method may not be suitable for studies involving sepsis patients with impaired kidney function.

Although the effect kidney function has on neopterin and total neopterin flux from plasma to urine is significant, there is no significant difference between eGFR for nonsepsis or sepsis patients (Fig. $4 \mathrm{~A}$ ). This indicates that the 
increased levels of seen in sepsis versus non-sepsis, is a real effect and not an artefact created by differences in eGFR. Furthermore, with kidney function having such a large effect on the levels of these biomarkers in plasma and urine, it would be recommended to take eGFR into account when assessing trauma using these biomarkers, particularly when there is any suspicion of kidney dysfunction.

\section{Conclusions}

Patients in ICU that were afflicted with sepsis experienced a greater level of oxidative stress and inflammation (shown by increased neopterin and total neopterin, IL-6 and CRP, respectively) than non-sepsis patients that were experiencing a similar level of trauma and organ dysfunction as determined by various trauma assessment scores. Over the 96 hour study period the levels of neopterin and total neopterin equalised between the groups. Kidney function, measured by glomerular filtration rate, has a moderate effect on neopterin and a large effect on 7,8-dihydroneopterin presence in the urine compared to plasma, however, this did not skew the increase in biomarker levels. Given this effect, when quantifying urinary or plasma neopterin and total neopterin it is important to take kidney function into consideration, as it has the potential to skew results.

Acknowledgements: We would like to acknowledge Intensive Care Research staff Jan Mahrtens, Anna Morris, Kim Parker and Emmeline Minto at Christchurch Hospital, New Zealand for their assistance in patient recruitment, sample collections and clinical information. This study was funded by the Intensive Care Foundation Grant (111595.01.p.QN) and the University of Otago (111595.01.p.QN), Christchurch, New Zealand and School of biological Sciences University of Canterbury student research support. Mr Gregory Baxter-Parker was supported by a University of Canterbury Doctoral Scholarship.

Conflict of interest: Prof. Gieseg is the member of Pteridines Editorial Board.

\section{References}

1. Nguyen HB, Rivers EP, Abrahamian FM, Moran GJ, Abraham E, Trzeciak S, et al.; Emergency Department Sepsis Education Program and Strategies to Improve Survival (ED-SEPSIS)
Working Group. Severe sepsis and septic shock: review of the literature and emergency department management guidelines. Ann Emerg Med. 2006 Jul;48(1):28-54.

2. Gyawali B, Ramakrishna K, Dhamoon AS. Sepsis: the evolution in definition, pathophysiology, and management. SAGE Open Med. 2019 Mar;7:2050312119835043.

3. Dellinger RP, Levy MM, Rhodes A, Annane D, Gerlach H, Opal SM, et al.; Surviving Sepsis Campaign Guidelines Committee including The Pediatric Subgroup. Surviving Sepsis Campaign: international guidelines for management of severe sepsis and septic shock, 2012. Intensive Care Med. 2013 Feb;39(2):165228.

4. Tasdelen Fisgin N, Aliyazicioglu Y, Tanyel E, Coban AY, Ulger F, Zivalioglu $\mathrm{M}$, et al. The value of neopterin and procalcitonin in patients with sepsis. South Med J. 2010 Mar;103(3):216-9.

5. Henriquez-Camacho C, Losa J. Biomarkers for sepsis. BioMed Res Int. 2014;2014:547818.

6. Uzzan B, Cohen R, Nicolas P, Cucherat M, Perret GY. Procalcitonin as a diagnostic test for sepsis in critically ill adults and after surgery or trauma: a systematic review and meta-analysis. Crit Care Med. 2006 Jul;34(7):1996-2003.

7. Damas P, Ledoux D, Nys M, Vrindts Y, De Groote D, Franchimont $P$, et al. Cytokine serum level during severe sepsis in human IL-6 as a marker of severity. Ann Surg. 1992 Apr;215(4):356-62.

8. Gaddam RR, Chambers S, Murdoch D, Shaw G, Bhatia M. Circulating levels of hydrogen sulfide and substance $P$ in patients with sepsis. J Infect. 2017 Oct;75(4):293-300.

9. Hensler T, Sauerland S, Lefering R, Nagelschmidt M, Bouillon $B$, Andermahr J, et al. The clinical value of procalcitonin and neopterin in predicting sepsis and organ failure after major trauma. Shock. 2003 Nov;20(5):420-6.

10. Ruokonen E, Ilkka L, Niskanen M, Takala J. Procalcitonin and neopterin as indicators of infection in critically ill patients. Acta Anaesthesiol Scand. 2002 Apr;46(4):398-404.

11. Baxter-Parker G, Prebble HM, Cross S, Steyn N, Shchepetkina $A$, Hock BD, et al. Neopterin formation through radical scavenging of superoxide by the macrophage synthesised antioxidant 7,8-dihydroneopterin. Free Radic Biol Med. 2020 Mar;152:142-51.

12. Gieseg SP, Maghzal G, Glubb D. Protection of erythrocytes by the macrophage synthesized antioxidant 7,8 dihydroneopterin. Free Radic Res. 2001 Feb;34(2):123-36.

13. Widner B, Mayr C, Wirleitner B, Fuchs D. Oxidation of 7,8-dihydroneopterin by hypochlorous acid yields neopterin. Biochem Biophys Res Commun. 2000 Aug;275(2):307-11.

14. Gieseg SP, Reibnegger G, Wächter H, Esterbauer H. 7,8 Dihydroneopterin inhibits low density lipoprotein oxidation in vitro. Evidence that this macrophage secreted pteridine is an anti-oxidant. Free Radic Res. 1995 Aug;23(2):123-36.

15. Wächter H, Fuchs D, Hausen A, Reibnegger G, Werner ER. Neopterin as marker for activation of cellular immunity: immunologic basis and clinical application. Adv Clin Chem. 1989;27:81-141.

16. Osse RJ, Fekkes D, Tulen JH, Wierdsma Al, Bogers AJ, van der Mast RC, et al. High preoperative plasma neopterin predicts delirium after cardiac surgery in older adults. J Am Geriatr Soc. 2012 Apr;60(4):661-8.

17. Gieseg SP, Baxter-Parker G, Lindsay A. Neopterin, Inflammation, and Oxidative Stress: What Could We Be Missing? Basel, Switzerland: Antioxidants; 2018. p. 7. 
18. Lindsay A, Janmale T, Draper N, Gieseg SP. Measurement of changes in urinary neopterin and total neopterin in body builders using SCX HPLC. Pteridines. 2014;25(2):53-62.

19. Fuchs D, Milstien S, Krämer A, Reibnegger G, Werner ER, Goedert JJ, et al. Urinary neopterin concentrations vs total neopterins for clinical utility. Clin Chem. 1989 Dec;35(12):2305-7.

20. Billiar TR. Nitric oxide. Novel biology with clinical relevance. Ann Surg. 1995 Apr;221(4):339-49.

21. Estelberger W, Weiss G, Petek W, Paletta B, Wächter H, Reibnegger $G$. Determination of renal clearance of neopterin by a pharmacokinetic approach. FEBS Lett. 1993 Aug;329(12):13-6.

22. Pecoits-Filho R, Heimbürger O, Bárány $P$, Suliman $M$, Fehrman Ekholm I, Lindholm B, et al. Associations between circulating inflammatory markers and residual renal function in CRF patients. Am J Kidney Dis. 2003 Jun;41(6):1212-8.

23. Goode HF, Cowley HC, Walker BE, Howdle PD, Webster NR. Decreased antioxidant status and increased lipid peroxidation in patients with septic shock and secondary organ dysfunction. Crit Care Med. 1995 Apr;23(4):646-51.

24. Goldberger BA, Loewenthal B, Darwin WD, Cone EJ. Intrasubject variation of creatinine and specific gravity measurements in consecutive urine specimens of heroin users. Clin Chem. 1995 Jan;41(1):116-7.

25. Alessio L, Berlin A, Dell'Orto A, Toffoletto F, Ghezzi I. Reliability of urinary creatinine as a parameter used to adjust values of urinary biological indicators. Int Arch Occup Environ Health. 1985;55(2):99-106.

26. Levey AS, Stevens LA, Schmid CH, Zhang YL, Castro AF 3rd, Feldman HI, et al.; CKD-EPI (Chronic Kidney Disease Epidemiology Collaboration). A new equation to estimate glomerular filtration rate. Ann Intern Med. 2009 May;150(9):604-12.

27. Davison AC, Hinkley DV. Bootstrap methods and their application. Cambridge university press; 1997 . https://doi. org/10.1017/CB09780511802843.

28. Werner ER, Bichler A, Daxenbichler G, Fuchs D, Fuith LC, Hausen $A$, et al. Determination of neopterin in serum and urine. Clin Chem. 1987 Jan;33(1):62-6.

29. Vincent JL, Moreno R, Takala J, Willatts S, De Mendonça A, Bruining $\mathrm{H}$, et al. The SOFA (Sepsis-related Organ Failure Assessment) score to describe organ dysfunction/failure. Intensive Care Med. 1996 Jul;22(7):707-10.

30. Vincent JL, de Mendonça A, Cantraine F, Moreno R, Takala J, Suter PM, et al. Use of the SOFA score to assess the incidence of organ dysfunction/failure in intensive care units: results of a multicenter, prospective study. Working group on "sepsisrelated problems" of the European Society of Intensive Care Medicine. Crit Care Med. 1998 Nov;26(11):1793-800.

31. Knaus WA, Draper EA, Wagner DP, Zimmerman JE. APACHE II: a severity of disease classification system. Crit Care Med. 1985 Oct;13(10):818-29.

32. Knaus WA, Wagner DP, Draper EA, Zimmerman JE, Bergner M, Bastos PG, et al. The APACHE III prognostic system. Risk prediction of hospital mortality for critically ill hospitalized adults. Chest. 1991 Dec;100(6):1619-36.

33. Baxter-Parker G, Roffe L, Cross S, Frampton C, Hooper GJ, Gieseg SP. Knee replacement surgery significantly elevates the urinary inflammatory biomarkers neopterin and 7,8-dihydroneopterin. Clin Biochem. 2019 Jan;63:39-45.

34. Baydar T, Yuksel O, Sahin TT, Dikmen K, Girgin G, Sipahi H, et al. Neopterin as a prognostic biomarker in intensive care unit patients. J Crit Care. 2009 Sep;24(3):318-21.

35. Faist E, Storck M, Hültner L, Redl H, Ertel W, Walz A, et al. Functional analysis of monocyte activity through synthesis patterns of proinflammatory cytokines and neopterin in patients in surgical intensive care. Surgery. 1992 Sep;112(3):562-72.

36. Yao YM, Yu Y, Wang YP, Tian HM, Sheng ZY. Elevated serum neopterin level: its relation to endotoxaemia and sepsis in patients with major burns. Eur J Clin Invest. 1996 Mar;26(3):224-30.

37. Flavall EA, Crone EM, Moore GA, Gieseg SP. Dissociation of neopterin and 7,8-dihydroneopterin from plasma components before HPLC analysis. J Chromatogr B Analyt Technol Biomed Life Sci. 2008 Feb;863(1):167-71.

38. Baxter-Parker G, Chu A, Petocz P, Samman S, Gieseg SP. Simultaneous analysis of neopterin, kynurenine and tryptophan by amine-HPLC shows minor oxidative stress from short-term exhaustion exercise. Pteridines. 2019;30(1):21-32.

39. Haupt W, Hohenberger W, Mueller R, Klein P, Christou NV. Association between preoperative acute phase response and postoperative complications. Eur J Surg. 1997 Jan;163(1):3944.

40. Galley HF. Oxidative stress and mitochondrial dysfunction in sepsis. Br J Anaesth. 2011 Jul;107(1):57-64.

41. Brown KA, Brain SD, Pearson JD, Edgeworth JD, Lewis SM, Treacher DF. Neutrophils in development of multiple organ failure in sepsis. Lancet. 2006 Jul;368(9530):157-69.

42. Justice JM, Tanner MA, Myers PR. Endothelial cell regulation of nitric oxide production during hypoxia in coronary microvessels and epicardial arteries. J Cell Physiol. 2000 Mar;182(3):359-65.

43. Sheldon J, Riches PG, Soni N, Jurges E, Gore M, Dadian G, et al. Plasma neopterin as an adjunct to $C$-reactive protein in assessment of infection. Clin Chem. 1991 Dec;37(12):2038-42.

44. Giannoudis PV, Smith MR, Evans RT, Bellamy MC, Guillou PJ. Serum CRP and IL-6 levels after trauma. Not predictive of septic complications in 31 patients. Acta Orthop Scand. 1998 Apr;69(2):184-8.

45. Volanakis JE. Human C-reactive protein: expression, structure, and function. Mol Immunol. 2001 Aug;38(2-3):189-97.

46. Bauer J, Ganter U, Geiger T, Jacobshagen U, Hirano T, Matsuda T, et al. Regulation of interleukin-6 expression in cultured human blood monocytes and monocyte-derived macrophages. Blood. 1988 Oct;72(4):1134-40.

47. Huber C, Batchelor JR, Fuchs D, Hausen A, Lang A, Niederwieser $D$, et al. Immune response-associated production of neopterin. Release from macrophages primarily under control of interferon-gamma. J Exp Med. 1984 Jul;160(1):310-6.

48. Aulitzky WE, Tilg H, Niederwieser D, Riccabona G, Obendorf L, Margreiter R, et al. Comparison of serum neopterin levels and urinary neopterin excretion in renal allograft recipients. Clin Nephrol. 1988 May;29(5):248-52.

49. Ohzato H, Yoshizaki K, Nishimoto N, Ogata A, Tagoh H, Monden $M$, et al. Interleukin- 6 as a new indicator of inflammatory status: detection of serum levels of interleukin- 6 and C-reactive protein after surgery. Surgery. 1992 Feb;111(2):201-9. 
50. Adamik B, Kübler-Kielb J, Golebiowska B, Gamian A, Kübler A. Effect of sepsis and cardiac surgery with cardiopulmonary bypass on plasma level of nitric oxide metabolites, neopterin, and procalcitonin: correlation with mortality and postoperative complications. Intensive Care Med. 2000 Sep;26(9):1259-67.

51. Perrone RD, Madias NE, Levey AS. Serum creatinine as an index of renal function: new insights into old concepts. Clin Chem. 1992 Oct;38(10):1933-53.

52. Ozbek E. Induction of oxidative stress in kidney. Int J Nephrol. 2012;2012:465897. 